\title{
Impact of Visual Perception Restoration in Quality of Life in Early Childhood
}

\author{
Laís Fabbro ${ }^{1}$, Marcio Scuoteguazza-Filho ${ }^{2}$, Erika Kazue Moribe ${ }^{1}$, Laísa Rodrigues ${ }^{1}$, Isabella \\ Almeida Oliveira da Silva ${ }^{1}$, Richard Lucas Konichi-Dias ${ }^{1}$, Daniel Nishiwama Silva Muniz ${ }^{1}$, \\ Nádia Fabbro $^{1}$, Daniele Natália Pacharone Bertolini Bidinotto ${ }^{1,3}$, Lucas Tadeu Bidinotto ${ }^{1,3,4 *}$. \\ ${ }^{I}$ Barretos Schoolof Health Sciences, Dr Paulo Prata-FACISB, Barretos, São Paulo, Brazil \\ ${ }^{2}$ Barretos Medical Specialties Outpatient (AME), Barretos, São Paulo, Brazil \\ ${ }^{3}$ Molecular Oncology Research Center, Barretos Cancer Hospital, Barretos, São Paulo, Brazil \\ ${ }^{4}$ Department of Pathology, UNESP - Univ. Estadual Paulista, School of Medicine, Botucatu, São \\ Paulo, Brazil
}

*Corresponding Author: Lucas Tadeu Bidinotto, Barretos School of Health Sciences - FACISB, Avenida Loja Maçônica Renovadora Barretos/SP/Brazil.

\begin{abstract}
Vision is the most influential sense in the integration of human beings into the environment. The early identification of problems in health-related quality of life $(Q o L)$ in children with impaired visual acuity enables further intervention, which can decrease the prevalence of poor educational, occupational and social outcomes. Thus, this study has the objective of evaluating the impact of corrective lenses use on scholars aged 4 to 5 years' restoration of quality of life. A number of 111 schoolchildren were evaluated. QoL was assessed using Children's Visual Function Questionnaire (CVFQ), while visual acuity (VA) was assessed by Snellen test (ST). Scholars with VA change equal to or greater than 20/40 or difference between the two eyes of two or more ST lines were referred to the ophthalmologist. Five domains of the CVFQ (General health, Vision health, Competence, Personality and Family impact) of scholars who needed corrective glasses were compared to those who did not have any VA alteration. The scholars diagnosed with alterations in VA after the ophthalmological consultation received corrective glasses and, after 3 months, the CVFQ was reapplied. For the statistical evaluation, $t$-student test was used, considering statistically significant the comparisons at $P \unlhd 0.05$. We found 18 scholars with the need of corrective lenses. Before its use, they presented lower scores in Visual health, Competence and Familiar impact CVFQ subscales. After three months of corrective lenses the scores were restored to levels similar to the children who did not need the use. Interestingly, we found an increase in General health subscale of the children which used corrective glasses compared to those who did not need its use. In conclusion, there is a subpopulation of schoolchildren aged 4 and 5 who have impaired VA, interfering, even with QoL, and the use of corrective glasses can lead to the restoration of QoL related to visual function.
\end{abstract}

Keywords: Snellen test, Visual acuity, Quality of Life

\section{INTRODUCTION}

Vision is essential for the development of capacities on social interaction, assimilation and learning; therefore, it is the most influential sense in the integration of human beings into the environment. The importance of vision is evident in children's development, since vision promotes the socialization processes. Additionally, it facilitates the acquirement of skills of orientation and locomotion in a safe and autonomous way ${ }^{1}$.

Caring children's visual health is essential and must be performed through visual stimuli and anticipation of the main eye problems. Such problems are the $3 \mathrm{rd}$ most frequent cause of child health problems; importantly, there is an intense correlation of visual problems and school performance. Since 1970, the World Health Organization (WHO) has recommended the implementation of prevention programs in ocular health. Moreover, in association with the International Agency for the Prevention of Blindness (IAPB), WHO created the "VISION 2020: The Right to Sight program", which aims to reduce preventable blindness worldwide until 2020, especially among schoolchildren ${ }^{2}$.

In Brazil, about $59 \%$ of eye disorders are preventable or treatable ${ }^{3}$. However, only $10 \%$ of the children who begun their academic life participated in a previous eye examination ${ }^{4}$. It is assumed that 19 million children aged 0 to 15 
years old have visual changes, of which 1.4 million manifest irreversible blindness ${ }^{5}$. In addition, about $30 \%$ of the expenses related to visual impairment and blindness is attributable to those that occur in childhood, even though blindness in the pediatric age group is responsible for $4 \%$ of world blindness ${ }^{6}$. In general, approximately half of the world's childhood blindness is due to preventable causes $(15 \% \text { treatable and } 28 \% \text { preventable })^{7}$.

In general, visual changes begin to manifest at preschool age through difficulties with visual vocabulary, carrying out manual work and reading, in the case of hyperopia, or even rejecting other distant activities giving preference to reading, in the case of myopia ${ }^{8}$. Therefore, the early identification of problems in health-related quality of life (QoL) in children with impaired visual acuity enables further intervention, which can decrease the prevalence of poor educational, occupational and social outcomes ${ }^{9}$.

Currently, there are few studies that address the correlation of visual impairment and the scholars' quality of life. Thus, this study has the objective of evaluating the impact ofcorrective lenses' use on quality of life restoration of students from Municipal Centers for Early Childhood Education (MCECEs).

\section{MeTHODS}

A total of 111 schoolchildren aged 4 to 5 years were evaluated. A trial of visual acuity (VA) was assessed by Snellen test (ST).
Schoolchildren with VA change equal to or greater than 20/40 or difference between the two eyes of two or more ST lines were referred to the ophthalmologist, who performed further exams to determine the need of corrective glasses. Quality of Life (QoL) was assessed using the Children's Visual Function Questionnaire (CVFQ), ${ }^{10}$ validated for Brazilian Portuguese, applied to their nurturers. Five domains of the CVFQ (General health, Vision health, Competence, Personality and Family impact) were analyzed. QoL of the students who needed corrective glasses was compared to those who did not have any VA alteration. The scholars diagnosed with alterations in VA after the ophthalmological consultation received corrective glasses and, after 3 months of use, the CVFQ was reapplied to the nurturers. Student's t-test was used, considering the comparisons to be statistically significant at $\mathrm{P} \leq 0.05$, using SPSS Statistics software v.25.

This work was approved by Barretos Cancer Hospital Ethics Committee (number 1343/2017)

\section{RESULTS}

A total of 111 children were analyzed, being 49 $(44.1 \%)$ female and $62(55.9 \%)$ male. The majority of nurturers were father/mother $(87.4 \%)$, ranged from 21 to 40 years old $(76.7 \%)$, white $(50.5 \%)$, married $(60.4 \%)$ (Table 1). After Snellen test screening, 18 scholars $(16.2 \%)$ were referred to ophthalmologist consultation and needed the use of corrective glasses.

Table1. Characteristics of the schoolchildren and their nurturers according to the need of the use of corrective lenses.

\begin{tabular}{|c|c|c|c|c|c|}
\hline & & \multicolumn{3}{|c|}{ Treatment } & \multirow[b]{2}{*}{$\mathrm{P}$ value } \\
\hline & & $\mathrm{N}$ & No & Yes & \\
\hline \multirow[t]{2}{*}{ Gender } & Female & 49 & $38(77.6 \%)$ & $11(22.4 \%)$ & 0.113 \\
\hline & Male & 62 & $55(88.7 \%)$ & $7(11.3 \%)$ & \\
\hline \multirow[t]{4}{*}{ Nurturer } & Father/Mother & 90 & $74(82.2 \%)$ & $16(17.8 \%)$ & 0.685 \\
\hline & Legal nurturer & 6 & $5(83.3 \%)$ & $1(16.7 \%)$ & \\
\hline & Grandparents & 5 & $5(100 \%)$ & $0(0 \%)$ & \\
\hline & Other & 2 & $2(100 \%)$ & $0(0 \%)$ & \\
\hline \multirow[t]{2}{*}{ Nurturergender } & Female & 87 & $72(82.8 \%)$ & $15(17.2 \%)$ & 0.687 \\
\hline & Male & 13 & $12(92.3 \%)$ & $1(7.7 \%)$ & \\
\hline \multirow[t]{5}{*}{ Nurturer age } & Lessthan 21yo & 6 & $5(83.3 \%)$ & $1(16.7 \%)$ & 0.874 \\
\hline & 21 to $30 y o$ & 43 & $36(83.7 \%)$ & $7(16.3 \%)$ & \\
\hline & 31 to 40 yo & 36 & $29(80.6 \%)$ & $7(19.4 \%)$ & \\
\hline & 41 to 50 yо & 13 & $11(84.6 \%)$ & $2(15.4 \%)$ & \\
\hline & More than 50yo & 5 & $5(100 \%)$ & $0(0 \%)$ & \\
\hline \multirow[t]{3}{*}{ Race } & White & 52 & $45(86.5 \%)$ & $7(13.5 \%)$ & 0.602 \\
\hline & Black & 17 & $15(88.2 \%)$ & $2(11.8 \%)$ & \\
\hline & Brown & 34 & $27(79.4 \%)$ & $7(20.6 \%)$ & \\
\hline \multirow[t]{4}{*}{ Matrial status } & Single & 31 & $25(80.6 \%)$ & $6(19.4 \%)$ & 0.731 \\
\hline & Married & 61 & $50(82.0 \%)$ & $11(18.0 \%)$ & \\
\hline & Divorced & 8 & $8(100 \%)$ & $0(0 \%)$ & \\
\hline & Widow & 1 & $1(100 \%)$ & $0(0 \%)$ & \\
\hline
\end{tabular}


Considering the 5 domains of CVFQ, we found lower score of Vision health of the scholars who actually needed the use of corrective glasses $(\mathrm{P}=0.032$, Figure 1A), prior the treatment. Additionally, there was a trend of decrease of
Competence $(\mathrm{P}=0.076)$ and Family impact $(\mathrm{P}=0.076)$ subscale scores. There were no differences in General health $(\mathrm{P}=0.573)$ and Personality $(\mathrm{P}=0.555)$ subscale scores (Figure 1A).
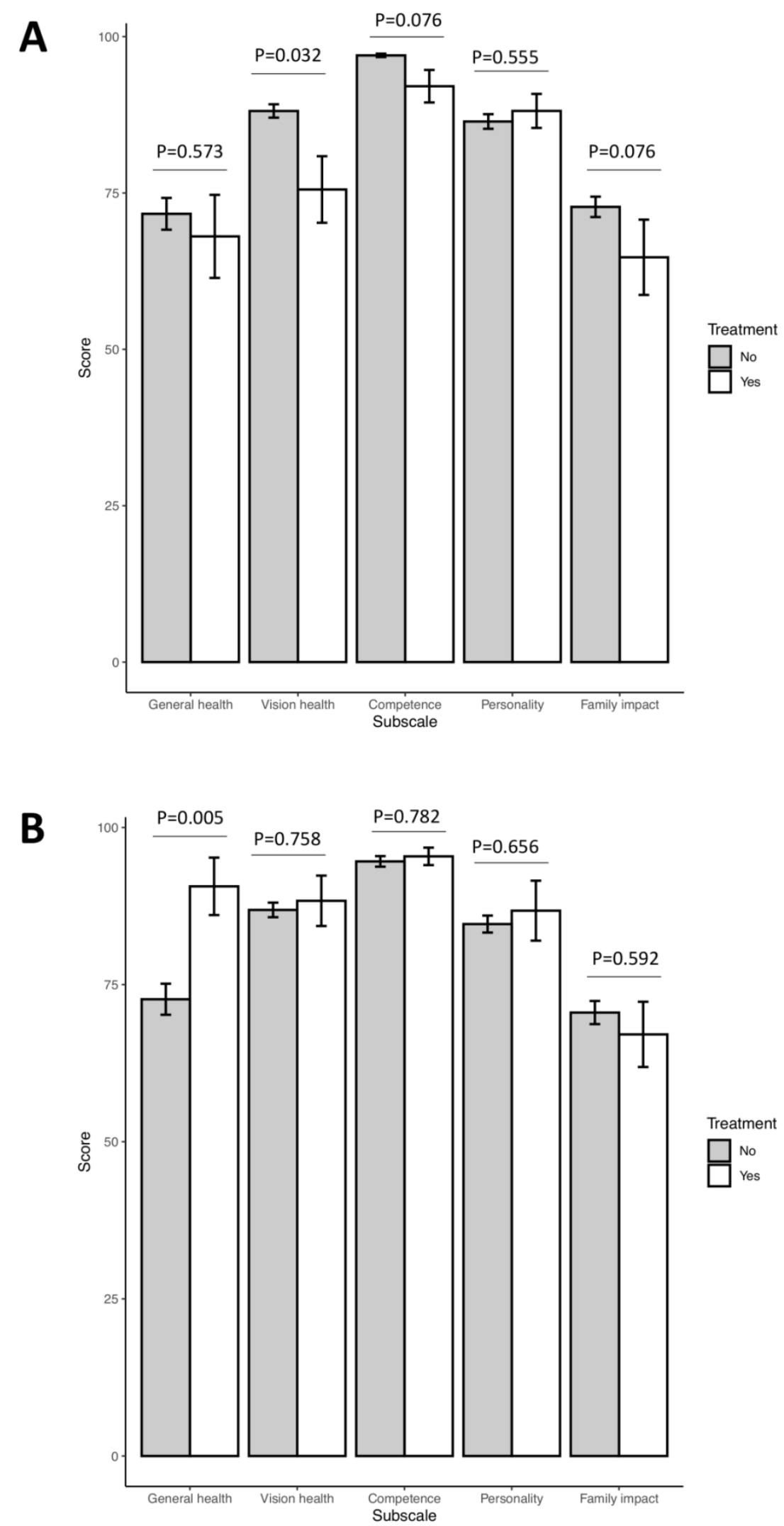

Figure1. Average scores in each Children's Visual Function Questionnaire subscale (A) prior the use of corrective lenses and $(B)$ three months after the use of corrective lenses. 
CVFQ was reapplied after three months, and there were not find differences in Vision health $(\mathrm{P}=0.758)$, Competence $(\mathrm{P}=0.782)$, Personality $(\mathrm{P}=0.656)$ and Family impact $(\mathrm{P}=0.592)$ (Figure 1B). However, nurturers of the children who used corrective glasses reported an increase in General health compared to those who did not need the use of corrective glasses $(\mathrm{P}=0.005$, Figure 1B).

\section{DISCUSSION}

The present work aimed at evaluating the impact of visual health problems on quality of life of scholars, and the restoration of quality of life after the use of correcting glasses. We found lower quality of life (QoL) of the children who needed the use of corrective glasses, and the rehabilitation it the QoL scores 3 months after corrective lenses use.

The interference of chronic diseases in the social, physical and emotional domains of children can be provided through quality of life instruments 11. Attention to the concept of quality of life is relatively recent, based on new paradigms for actions and attitudes related to the rehabilitation sectors ${ }^{12}$. Quality of life is defined as "an individual's perception of their position in life in the context of the culture and value systems in which they live and in relation to their goals, expectations, standards and concerns" ${ }^{13}$. When providing results through specific questionnaires, the assessment of quality of life allows comparisons between children with or without co morbidities ${ }^{14}$. As a way of assessing the impact of impaired visual acuity on children and their families, the Children's Visual Function Questionnaire (CVFQ) is an important tool. The incorporation of CVFQ in pediatric eye clinical studies contributes to a more comprehensive understanding of treatment results, both in terms of possible direct benefits for patients and their families ${ }^{15}$.

We found that the children who needed the use of corrective glasses presented lower scores in Vision health, Competence and Family impact. Competence subscale addresses common activities of daily living, such as the recognizing faces, playing outdoor games or brushing teeth. Family impact subscale addresses the nurturers' concerns with the child's vision ${ }^{16}$. Therefore, the results show that, besides the nurturers present insights and concerns about possible visual function problems of the children, they did not take the children to a consultation with an ophthalmologist. It is important to emphasize that three months after the use of corrective lenses, the scores in these subscales were restored to the same levels of the children who did not have any visual function problem. Similar results were found to the concern of use of corrective lenses, which revealed that the QoL reported by those responsible for children who used corrective lenses was worse than the parents of children who did not wear glasses ${ }^{17}$. We found higher values in General health subscale of the scholars who used the corrective lenses for three months. However, since the data are obtained from the parents, there is some difficulty in effectively evaluating the children's quality of life, and studies indicate that the parents' and children's reports sometimes does not coincide ${ }^{18}$. Considering that General health subscale has only one question, it is possible that this data was biased by nurturer's response.

In this study, we found that about $13 \%$ of the children had any visual problem not previously reported, emphasizing the importance of screening activities since the beginning of scholar age. The concentration of regular visual screening activities takes place in high-income countries. In Ohio, USA, for example, children are screened in kindergarten; in Sweden, visual acuity is measured in children of preschool age and again at seven and 10 years of age. In the United Kingdom, visual screening is recommended only for children aged four to five years ${ }^{19}$. Although screening programs are incorporated in low-income countries, a large portion of children do not undergo eye exams, especially when referring to rural areas ${ }^{20}$.Thus, it is unquestionable the relevance of eye health campaigns, since they can prevent and treat visual changes, thus enhancing the current and future quality of life for children, both in developed countries and in countries under development. Public policies for this purpose must be maintained and their functionality must be guaranteed by the competent organs.

\section{ACKNOWLEDGEMENTS}

Laís Fabbro was funded by Fundação de Amparo a Pesquisa do Estado de São Paulo (FAPESP) fellowship number 2017/02935-8.

\section{REFERENCES}

[1] Ciampo LAD, Cardoso AL, Nascimento CFG, Teles LPM, Mendonça CQ, Ferraz I, Costa R, Pereira CFA. Reduced visual acuity screening in a Primary Care Unit. Rev Bras Oftalmol. 2019;78(4): 250-254.

[2] Becker TOF, Miura H, Cortela DCB. Evaluation of visual acuity in municipal 
elementary school students. Rev Bras Oftalmol. 2019; 78(1): 37-41.

[3] Brito PR, Veitzman S. Causes of blindness and low vision in children. Arq BrasOftalmol. 2000; 63(1): 49-54

[4] Silva CMF, Almeida DR, Bernardes RR, Bazzano FCO, Mesquita FM, Magalhães CHT et al. School performance: visual acuity interference. Rev BrasOftalmol. 2013; 72 (3): 168-171.

[5] Elsman EBM, Baaj MA, Rens GHMB, Sijbrandi W, Broek EGCVD; Schakel W; et al. Interventions to improve functioning, participation, and quality of life in children with visual impairment: a systematic review. Survey of Ophthalmology.2019;64(4): 512-557.

[6] Cochrane GM, Marella M, Keeffe JE, Lamoureux EL. The Impact of Vision Impairment for Children (IVI_C): Validation of a Vision-Specific Pediatric Quality-of-Life Questionnaire Using Rasch Analysis. Investigative Ophthalmology \& Visual Science. 2011; 52(3): 1632-1640.

[7] Brazilian Ophthalmology Council. Conditions of Eye Health in Brazil; 2019. Available from: http://www.cbo.com.br/novo/publicacoes/condi coes_saude_ocular_brasil2019.pdf [Accessed 8th April 2020].

[8] Fernandes MA. The implications of visual problems in the process children's school learning. Available from: https://ubibliorum. ubi.pt/bitstream/10400.6/1216/1/Disserta\%C3\%A 7\%C3\%A3o\%20Mariana\%20Fernandes.pdf [Accessed 8th April 2020].

[9] Gerson CA, Wentz A, Abraham AG, Mendley SR, Hooper SR, Butler RW, et al. HealthRelated Quality of Life of Children with Mild to Moderate Chronic Kidney Disease Pediatrics. 2010;125(2): 349-357.

[10] Bestilleiro LMC, Rios SS, Berezovsky A, Tartarella MB. Assessing vision-related quality of life in children with bilateral congenital cataracts. Arq Bras Oftalmol. 2009; 72(4): 467480.

[11] Roncada C, Mattiello R, Pitres PM, Sarria EE. Specific instruments to assess quality of life in children and adolescents with asthma. Jornal de Pediatria. 2013;89 (3): 217-225.

[12] Lopes MCB. Relationship between the Children's Visual Function Questionnaire and psychophysical measures of visual accuity and chromaticity discrimination in visually impaired children. Available from: https: //www.teses.usp.br/teses/disponiveis/47/47135/ tde-17032015-121437/publico/lopes_original. pdf [Accessed 5th April 2020].

[13] WHO. Study protocol for the World Health Organization project to develop a Quality of Life assessment instrument (WHOQOL). Qual Life Res. 1993;2(2):153-159.

[14] Schepers SA, van Oers HA, Maurice-Stam H et al. Health related quality of life in Dutch infants, toddlers, and young children. Health Qual Life Outcomes. 2017;81(15).

[15] Birch EE, Cheng CS, Felius J. Validity and reliability of the Children's Visual Function Questionnaire (CVFQ). J AAPOS. 2007;11 (5): 473-479.

[16] Avery RA, Hardy KK. Vision specific quality of life in children with optic pathway gliomas. J Neurooncol. 2014;116(2):341-347.

[17] Yamada T, Hatt SR, Leske DA, Holmes JM. Spectacle wear in children reduces parental health-related quality of life. J AAPOS. 2011;15(1):24-28.

[18] Boulton M, Haines L, Smith D, et al. Healthrelated quality of life of children with vision impairment or blindness. Developmental Medicine \& Child Neurology.2006;48(8): 656-61.

[19] Public Health England (PHE). New guidance to improve vision screening for young children. Available from:www.gov. uk/government/news /new-guidance-to-improve-vision-screening-for -young-children [Accessed 3th April 2020]

[20] Congdon N, Zheng M, Sharma A, Choi K, Song Y, Zhang $\mathrm{M}$, et al. Prevalence and determinants of spectacle nonwear among rural Chinese secondary schoolchildren: the Xichang Pediatric Refractive Error Study Report 3. Archives of Ophthalmology. 2008; 126 (12): 1717-1723.

Citation: Laís Fabbro et.al, "Impact of Visual Perception Restoration in Quality of Life in Early Childhood”, International Journal of Research Studies in Medical and Health Sciences. 2020; 5(5): 15-19.

Copyright: (c) 2020 Laís Fabbro et.al., This is an open-access article distributed under the terms of the Creative Commons Attribution License, which permits unrestricted use, distribution, and reproduction in any medium, provided the original author and source are credited. 